\title{
Influencia de la relación Ti/N sobre la resistencia a la deformación en caliente y sobre la recristalización estática en aceros estructurales
}

\author{
M. I. VEGA, S.F. MEDINA, A. QUISPE, M. GÓMEZ \\ Centro Nacional de Investigaciones Metalúrgicas (CENIM-CSIC), 28005-Madrid
}

\begin{abstract}
Para conseguir una disolución completa de los nitruros de titanio (TiN) es necesario alcanzar temperaturas próximas a la temperatura de fusión o incluso temperaturas superiores correspondientes al estado líquido. Esto hace que los TiN sean buenos controladores del tamaño de grano austenítico. Esta característica es aprovechada en los aceros estructurales para controlar la evolución de la microestructura en todo el proceso de laminación en caliente y en procesos de aporte de calor como la soldadura en la zona afectada térmicamente (ZAT). Sin embargo, es prácticamente desconocida la influencia cuantitativa que ejercen dichas partículas sobre la resistencia en caliente y sobre la cinética de la recristalización estática, responsable esta última de la evolución microestructural durante la laminación en caliente. En el presente trabajo se ha estudiado la influencia de diferentes composiciones de Ti y N sobre la resistencia en caliente, determinando la tensión pico de las curvas de fluencia y la energía de activación. La influencia sobre la recristalización estática se ha determinado mediante las curvas de fracción recristalizada frente al tiempo y con la energía de activación. Para comprender la influencia de la relación Ti/ $\mathrm{N}$ de los aceros se realizó un análisis de los precipitados mediante Microscopía Electrónica de Barrido y de Transmisión.
\end{abstract}

Palabras clave: Relación Ti/N, tensión máxima; recristalización estática; estado de precipitación

\section{Influence of the $\mathrm{Ti} / \mathrm{N}$ ratio on the strength in the hot deformation and on the static recrystallisation in structural steels}

To obtain a complete dissolution of the nitrides of titanium (TiN) is necessary to reach temperatures close to the melting temperature or even higher temperatures corresponding to the liquid state. For this reason, TiN are good controllers of the austenitic grain size. This characteristic is used in structural steels to control the microestructural evolution in the hot rolling and in heat processes like in the welding in the zone affected thermically (ZAT). However, the quantitative influence of TiN particles on the hot deformation strength and on the static recrystallisation, this latter responsible for the microestructural evolution, is practically unknown. In the present work, the influence of different Ti and $\mathrm{N}$ compositions on the hot deformation strength have been studied determining the peak stress of the flow curves and the activation energy. The influence on the static recrystallization have been determined by means of the static recrystallisation fraction versus time and with the activation energy. A precipitate study by means of SEM and TEM was done in order to better understand the influence of the Ti/ $\mathrm{N}$ ratio of the steels.

Keywords: Ti/N ratio, peak stress, static recrystallisation, precipitation state

\section{INTRODUCCIÓN}

Los nitruros de titanio (TiN) son muy poco solubles en la austenita $(1,2)$. Esta característica hace del Ti y N elementos ideales para controlar el tamaño de grano austenítico. Por ello, su adición es indispensable en aceros estructurales con requisitos de soldabilidad. Además de poseer buenas características de soldabilidad, los aceros estructurales deben presentar también buenos niveles de resistencia y tenacidad. Para conseguir esto es necesario una microestructura final de grano fino por lo que es necesario influir sobre la evolución microestructural de la austenita durante la deformación en caliente desde el horno de recalentamiento hasta el final de la laminación.

El papel de las partículas TiN en el control del tamaño de grano austenítico puede explicarse e interpretarse mediante las expresiones de Zener y Gladman $(3,4)$ que relacionan tamaño de precipitado, fracción precipitada y tamaño de grano austenítico. Los resultados existentes coinciden en que una relación Ti/ $\mathrm{N}$ entre 1 y 3 proporciona buenos resultados en el control del tamaño de grano (5-8).

Por otra parte, es conocido que la recristalización estática experimentada por la austenita entre pasos, en la laminación en caliente, está influenciada por todas las variables que intervienen en la laminación: temperatura, deformación, velocidad de deformación $(9,10)$. Sin embargo, se desconoce prácticamente la influencia cuantitativa de la relación $\mathrm{Ti} / \mathrm{N}$ o su correspondiente estado de precipitación.

\section{PROCEDIMIENTO EXPERIMENTAL}

Se fabricaron seis aceros en un horno de inducción al vacío a partir de hierro electrolítico de alta pureza. Los aceros pueden clasificarse en dos grupos (Tabla I) de acuerdo al contenido de nitrógeno. En un grupo hay tres aceros con 40 ppm de nitrógeno (S1, S2, S3) y en el otro grupo otros tres aceros con 80 ppm de nitrógeno (S4, S5, S6). Los aceros S1 y $\mathrm{S} 4$ no se alearon con titanio para utilizarlos de referencia.

TABLA I. COMPOSICIÓN QUÍMICA DE LOS ACEROS FABRICADOS

\begin{tabular}{|cccccccccc|}
\hline Acero & $\mathbf{C}$ & $\mathbf{M n}$ & $\mathbf{S i}$ & $\mathbf{P}$ & $\mathbf{S}$ & $\mathbf{T i}$ & $\mathbf{A l}$ & $\mathbf{N}_{\mathbf{2}}$ & $\mathbf{O}_{\mathbf{2}}$ \\
\hline $\mathbf{S 1}$ & 0,12 & 1,25 & 0,34 & 0,014 & 0,015 & 0 & 0,047 & 0,004 & 0,001 \\
\hline S2 & 0,12 & 1,23 & 0,32 & 0,011 & 0,013 & 0,021 & 0,045 & 0,0046 & 0,0006 \\
\hline S3 & 0,12 & 1,16 & 0,31 & 0,011 & 0,013 & 0,047 & 0,045 & 0,0043 & 0,0007 \\
\hline S4 & 0,1 & 1,13 & 0,3 & 0,007 & 0,012 & 0 & 0,032 & 0,008 & 0,0013 \\
\hline S5 & 0,12 & 1,18 & 0,29 & 0,007 & 0,012 & 0,018 & 0,034 & 0,008 & 0,0011 \\
\hline S6 & 0,11 & 1,09 & 0,25 & 0,005 & 0,012 & 0,031 & 0,035 & 0,0083 & 0,0011 \\
\hline
\end{tabular}

Los ensayos de fluencia se llevaron a cabo mediante una máquina de torsión. Se realizaron dos tipos de ensayo. Para la obtención de 
las curvas de fluencia la probeta se calienta inicialmente hasta una temperatura de austenización de $1300^{\circ} \mathrm{C}$ durante 10 minutos. Luego la temperatura disminuye hasta la temperatura del ensayo 900, 1000 ó $1100^{\circ} \mathrm{C}$ y se aplica una deformación equivalente de 2,7 , suficiente para producir la recristalización dinámica del acero. Para determinar la fracción recristalizada frente al tiempo se aplicó la técnica de la "doble deformación", en particular el método conocido por "back extrapolation" (11). Se aplicaron dos temperaturas de austenización: $1300^{\circ} \mathrm{C}$ y $1100{ }^{\circ} \mathrm{C}$ durante 10 minutos respectivamente. El ensayo se realiza enfriando rápidamente desde la temperatura de austenización hasta la temperatura de deformación: $900,1000,1100{ }^{\circ} \mathrm{C}$. Se estudiaron dos valores de deformación, concretamente 0,2 y 0,35 , ambos inferiores a la deformación pico para evitar la recristalización dinámica. Las velocidades de deformación seleccionadas fueron 1,451 ó 3,628 s-1.

El estudio de los precipitados más finos se realizó mediante Microscopía Electrónica de Transmisión (TEM) empleando la técnica de extracción por réplica de carbono. Los precipitados más gruesos fueron estudiados por Microscopía Electrónica de Barrido (SEM). Los estados de precipitación estudiados correspondían a las temperaturas de $1100^{\circ} \mathrm{C}$ y $1300^{\circ} \mathrm{C}$, respectivamente.

\section{RESULTADOS Y DISCUSIÓN}

\subsection{Análisis de los precipitados}

La identificación de los precipitados como TiN se realizó mediante difracción de electrones y análisis de dispersión de rayos X. Por otra parte, se observó que existía una distribución de tamaños que variaba entre unos pocos nanómetros hasta micrómetros. Por ello, fue necesario establecer una división de precipitados en dos grupos, finos (tamaño $<100 \mathrm{~nm}$ ) y gruesos (tamaño $>100 \mathrm{~nm}$ ). El estado de precipitación estudiado fue el correspondiente a $1300^{\circ} \mathrm{C}$ en todos los aceros y en los aceros S5 y S6 se estudió también a $1100^{\circ} \mathrm{C}$. En la figura 1 se muestra una micrografía realizada en el TEM del estado de precipitación del acero $\mathrm{S} 5$ a $1300^{\circ} \mathrm{C}$. Como ejemplo, en la figura 2 se representa la distribución de precipitados finos de dicho acero a $1300^{\circ} \mathrm{C}$. En la Tabla II se indica el tamaño medio ponderado de los precipitados finos y gruesos, respectivamente, para cada acero.

TABLA II. TAMAÑO MEDIO DE PRECIPITADO

\begin{tabular}{|l|c|c|c|c|c|c|}
\hline \multicolumn{1}{|c|}{ Aceros } & S2 & S3 & \multicolumn{2}{c|}{ S5 } & \multicolumn{2}{c|}{ S6 } \\
\hline Ratio Ti/N & 4,56 & 10,93 & \multicolumn{2}{c|}{2,25} & \multicolumn{2}{c|}{3,73} \\
\hline Temperatura, ${ }^{\circ} \mathbf{C}$ & 1300 & 1300 & 1300 & 1100 & 1300 & 1100 \\
\hline Precipitados finos, nm & 23 & 65,2 & 13,8 & 6,5 & 14,1 & 7,6 \\
\hline Precipitados gruesos, $\mu \mathrm{m}$ & 2,18 & 2,57 & 1,26 & 0,41 & 2,32 & 1,35 \\
\hline
\end{tabular}

\subsection{Resistencia a la deformación en caliente. Influencia de la relación $\mathrm{Ti} / \mathrm{N}$}

En la figura 3 puede verse un ejemplo de las curvas tensión-deformación (curvas de fluencia) de los aceros S4, S5 y S6 obtenidas para unas condiciones de ensayo determinadas. El valor máximo de la tensión, conocida como tensión pico, indica la resistencia en caliente del acero a ser deformado. Los aceros S5 y S6 son los que presentaron las mayores tensiones pico en todas las condiciones de deformación, lo que significa que estos aceros presentarán una mayor resistencia a deformarse en caliente y por tanto las fuerzas de laminación a aplicar deben ser mayores.

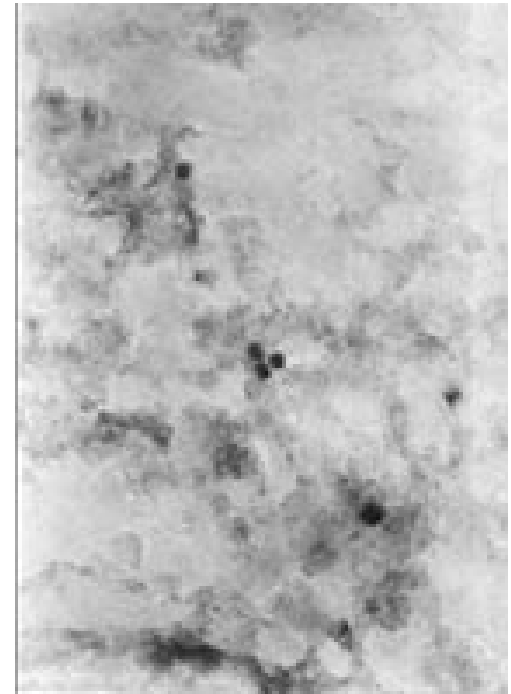

Figura 1. Imagen del TEM (x135000) Acero S5. Temple a $1300^{\circ} \mathrm{C}$

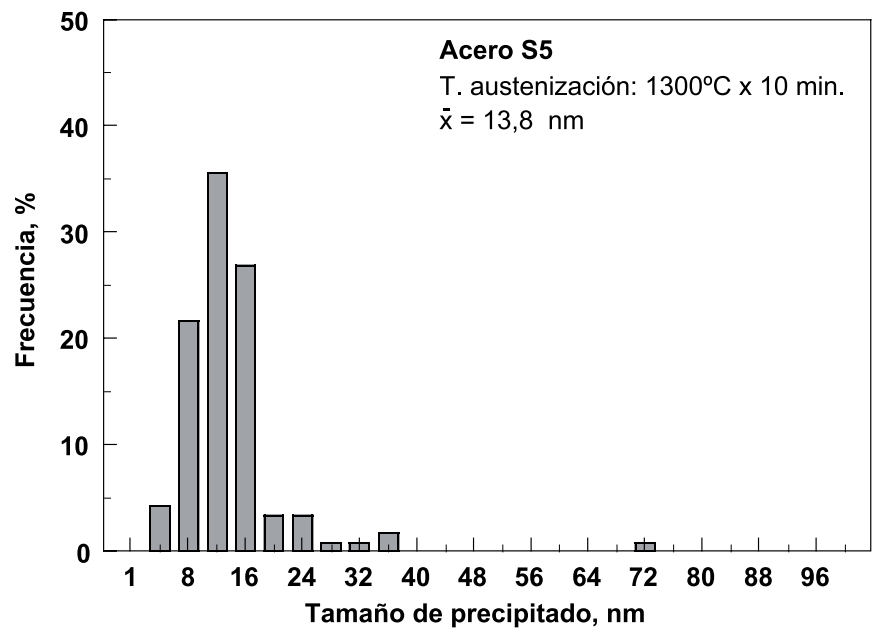

Figura 2. Distribución de precipitados finos en el acero S5 a $1300^{\circ} \mathrm{C}$

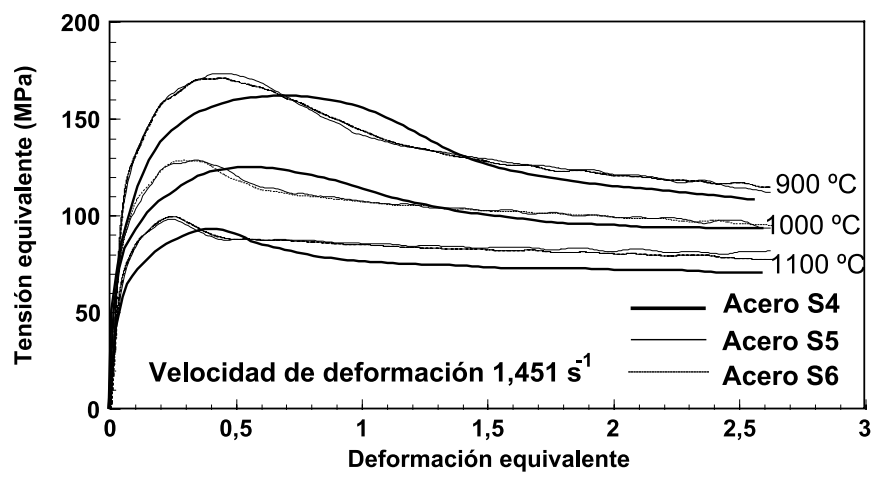

Figura 3. Curvas de fluencia de los aceros S4, S5 y S6.

En contra de lo que podría parecer más lógico, que a mayor contenido de elemento microaleante mayor es la resistencia, se ha visto que no sucede así. El acero 55 tiene el menor contenido de titanio y sin embargo presenta una resistencia a la deformación en caliente mayor que los otros aceros. Las razones hay que buscarlas en la relación Ti/N y en su correspondiente estado de precipitación.

Como puede verse, los aceros S5 y S6, con relaciones Ti/ N inferiores o más próximas a la estequiométrica $(3,42)$ y por tanto con los ta- 
maños medios de precipitados más pequeños tanto en la distribución de precipitados finos como en la de gruesos, son los que presentaron mayores tensiones dado que los precipitados ejercen una mayor influencia sobre la resistencia.

Otra forma de medir la influencia del Ti y el N sobre la resistencia a la deformación en caliente es mediante la energía de activación. Para ello, se representó en unos gráficos la diferencia de la energía de activación de cada acero determinada experimentalmente con respecto a su acero base frente a la relación Ti/N (figura 4). Como se observa, las energías de activación mayores corresponden a los aceros con relaciones más próximas a 2 . El acero S3 presenta una energía menor que el acero S1, probablemente debido a que las partículas bastas de este acero han servido de fuentes de nucleación de la recristalización dinámica, disminuyendo de este modo el valor de la tensión pico.

\subsection{Recristalización estática. Influencia de la relación $\mathrm{Ti} / \mathrm{N}$}

A partir de los ensayos isotérmicos descritos anteriormente y aplicando el método "back extrapolation", se obtuvieron las curvas de fracción recristalizada frente al tiempo. La figura 5 muestra un ejemplo correspondiente al acero S3. Como se observa, para la temperatura de $900^{\circ} \mathrm{C}$ se ha producido una meseta que indica una inhibición momentánea de la recristalización estática. Esta es debida a la precipitación inducida por la deformación de Ti y $\mathrm{N}$ que permanecían en solución a la temperatura de austenización $\left(1300^{\circ} \mathrm{C}\right)$. Esta inhibición solo se ha producido en algunas condiciones de formación en los aceros S3 y S6 con mayor contenido de titanio.

Para analizar la cinética de la recristalización estática se representó el tiempo que tarda en recristalizar el $50 \%$ del volumen $\left(\mathrm{t}_{0,5}\right)$ frente a la inversa de la temperatura (figura 6). Del estudio realizado se observa que todos los aceros, excepto el acero S3, tienen una cinética de recristalización estática más rápida que la del acero base correspondiente, a pesar de que en algunos casos, como en los aceros S5 y S6, el tamaño pequeño de los precipitados correspondientes tienden a retrasar o inhibir la cinética del proceso. La razón se debe al menor tamaño de grano de los aceros con titanio por la acción de control que ejercen los precipitados sobre el crecimiento de grano.

Para medir la influencia de la relación Ti/N sobre la recristalización se representó, del mismo modo que para la energía de activación para la deformación, la diferencia entre las energías de activación de cada acero y el acero base correspondiente frente a la relación Ti/N (figura 7).

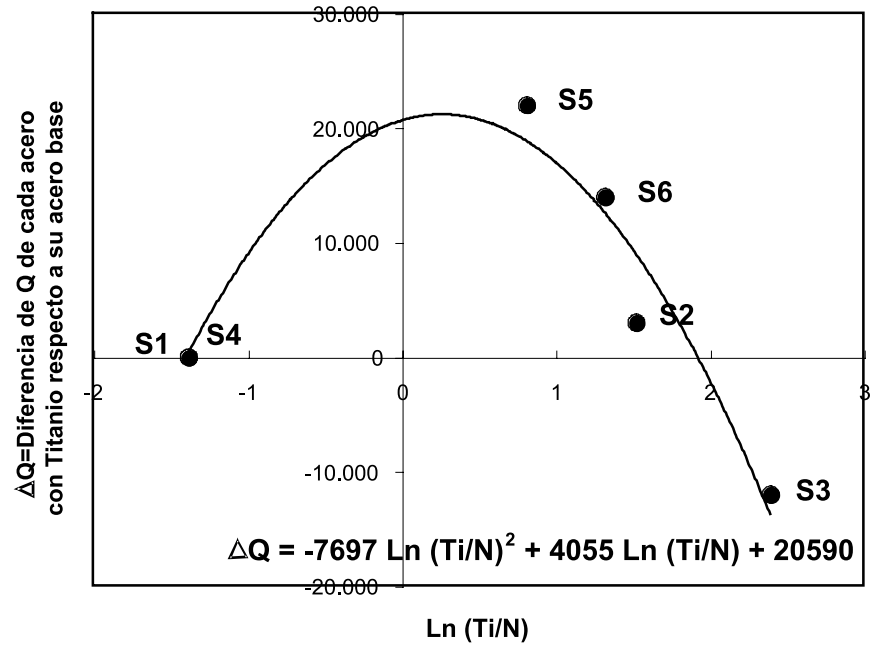

Figura 4. Influencia de la relación Ti/N sobre la energía de activación para la deformación (fluencia)

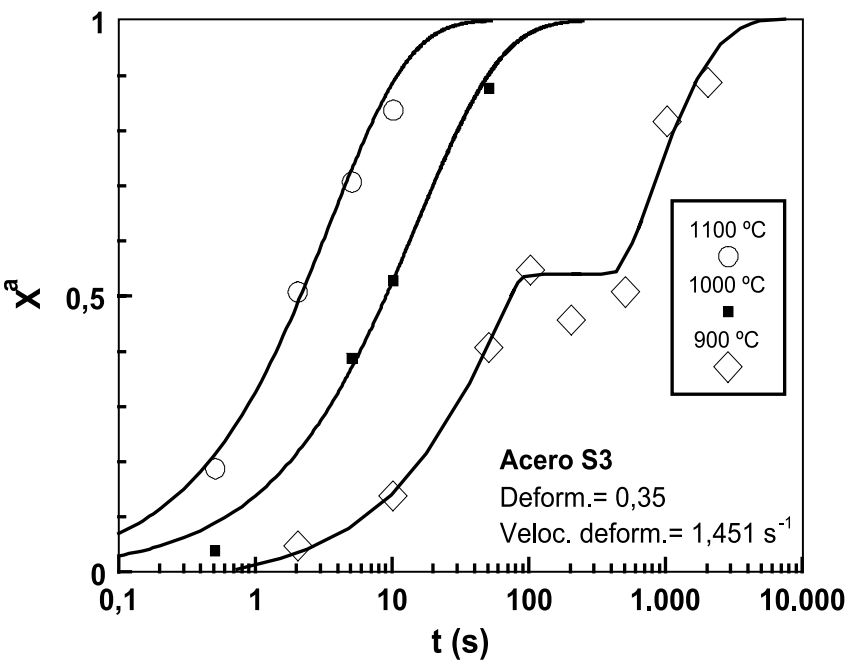

Figura 5. Curvas de fracción recristalizada frente al tiempo. Acero S3

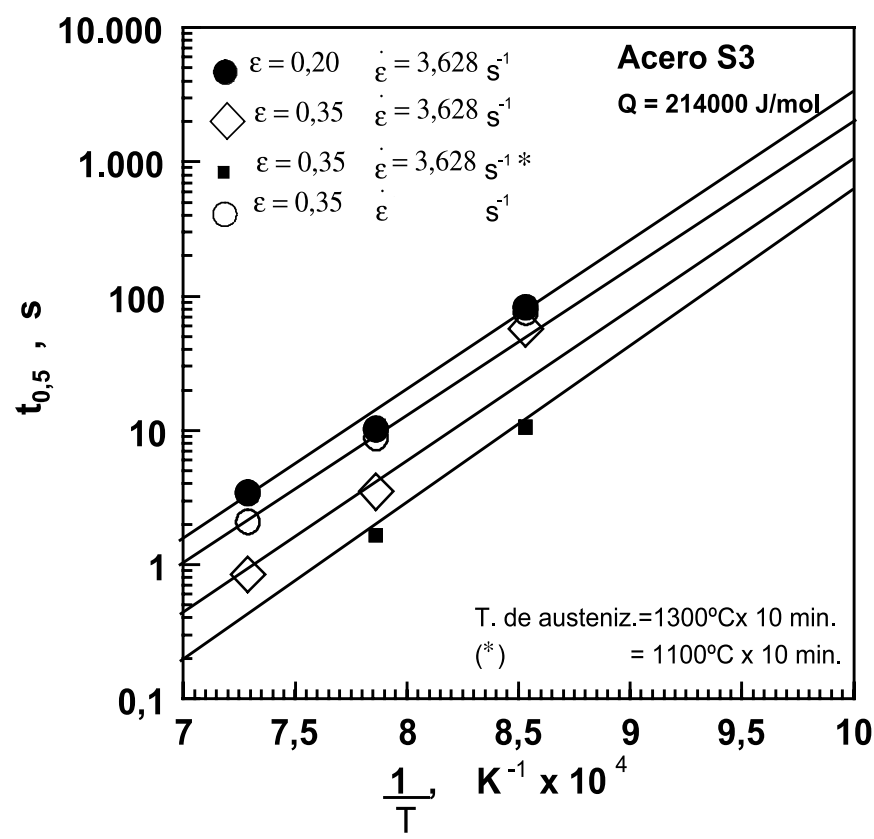

Figura 6. Tiempo que tarda en recristalizar el 50\% del acero S3 frente a la inversa de $\mathrm{T}(\mathrm{K})$.

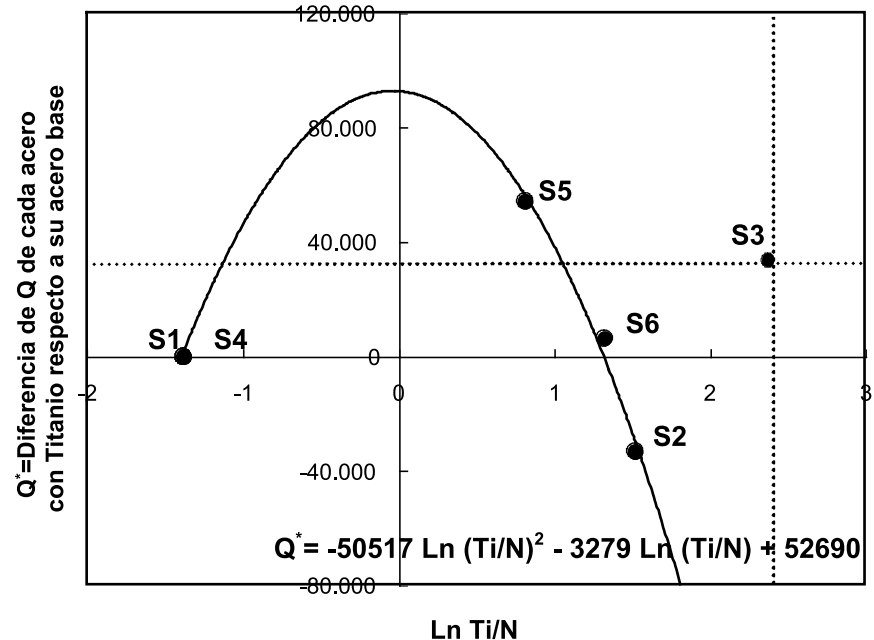

Figura 7. Influencia de la relación Ti/N sobre la energía de activación de la recristalización estática 
En principio podría pensarse que el acero S3 debería tener una energía de activación inferior a los otros aceros al tener una relación $\mathrm{Ti} / \mathrm{N}$ muy superior a la estequiométrica y por ello un tamaño medio de precipitado mayor que el resto de los aceros que no ejercen inhibición sobre la nucleación y crecimiento de los núcleos recristalizados y que además pueden favorecer dicho proceso debido al efecto de nucleación heterogénea. Sin embargo, el acero S3 es el acero con el mayor contenido de titanio y con más titanio en solución de todos los aceros que contribuye a retrasar la cinética de la recristalización. Dado que el titanio tiene un volumen atómico similar al del niobio es de esperar que también tenga una gran influencia sobre la energía de activación en la recristalización estática. Esto explicaría porqué el acero S3 tiene una energía de activación mayor.

Por tanto, no pudo obtenerse una expresión que relacionase la energía de activación correspondiente a la recristalización estática con la relación Ti/N. Esto nos indica que la energía de activación no solo depende de la relación $\mathrm{Ti} / \mathrm{N}$, es decir, del estado de precipitación, sino del titanio en solución. Los resultados obtenidos ponen de manifiesto que el mecanismo regulador del movimiento de dislocaciones durante la deformación en caliente de la austenita, es diferente a la autodifusión en límites de grano, mecanismo regulador de la recristalización estática, no sólo por el diferente valor de la energía de activación en ambos fenómenos sino también por el diferente efecto del titanio.

\section{CONCLUSIONES}

1. En los aceros microaleados un incremento del contenido de titanio no significa necesariamente que la resistencia a la deformación también aumente.

2. Se ha obtenido una relación entre el estado de precipitación y la resistencia máxima que presentan los aceros durante la deformación en caliente.

3. La energía de activación de la recristalización estática no depende solo del estado de precipitación sino del titanio en solución.
4. Una relación $\mathrm{Ti} / \mathrm{N}(\cong 2)$, capaz de controlar el tamaño de grano austenítico a altas temperaturas, no inhibe la recristalización estática en la misma medida. La causa más probable podría ser el cambio del estado de precipitación inicial durante la laminación.

\section{AGRADECIMIENTOS}

Nuestro agradecimiento al programa CECA de la unión europea por la financiación del trabajo realizado.

\section{BIBLIOGRAFÍA}

1. E.T. Turkdogan: «Causes and effects of nitride and carbonitride precipitation during continous casting». Iron Steelmaker: 3 [5] 61-75 (1989)

2. K. Narita: «Physical chemistry of the groups IVa (Ti, Zr), VA(V, Nb, Ta) and the rare earth elements in steel». Trans. Iron and Steel Inst. of Japan 15 [2] 145-152 (1975).

3. C.S. Smith: «Grain, Phases and Interfases: An Interpretation of Microstructure». Comunicación Privada de C. Zener a C.S. Smith. Citado en pág. 47. Trans. AIME 175 [15] 15-51 (1948).

4. T. Gladman: «On the Theory of the Effect of Precipitate Particles on Grain Growth in Metals». Proc. Royal Soc. 294 298-309 (1966).

5. P.E. Reynolds. «Effect of Titanium Treatment on Grain Size Control in Low Alloy Steels». ISIJ Int. 34 689-* (1994)

6. F.B. Pickering: «Overview of Titanium Microalloyed Steels» pp. 10-11 en Titanium Technology in Microalloyed Steels. The Institute of Materials, Londres (Inglaterra) 1997.

7. S. F. Medina y J.E. Mancilla. «The Influence of Titanium Recrystallization of Hot Deformed Austenite and on Induced Precipitation Kinetics». Scripta. Metall. Mater 30 [1] 73-78 (1994).

8. J.H. Beynon y C.M. Sellars: «Modelling Microstructure and Its Effects during Multipass Hot Rolling». ISIJ International, 32 [3] 359-367 (1992).

9. H.L. Andrade, M.G. Akben y J.J. Jonas: «Effect of Molybdenum, Niobium and Vanadium on Static Recovery and Recrystallization and on Solute Strengthening in Microalloyed Steels». Metallurgical Transactions A, 14 A 1967-1977 (1983).

Recibido: 1.2.03

Aceptado: 30.11 .03 